\title{
IRRATIONAL POWER SERIES
}

L. J. MORDELL

In a paper with the same name which is to appear in due course in the Proceedings of the American Mathematical Society, ${ }^{1}$ Dr. Morris Newman proves the

TheOREM. Let $\alpha$ be a real number and let $f(x)$ be a polynomial of degree $\geqq 1$. Then

$$
F(x)=\sum_{n=0}^{\infty} f([n \alpha]) x^{n}
$$

is a rational function of $x$ if and only if $\alpha$ is a rational number.

Here $[x]$ is the integer part of $x$. We write $x=[x]+\{x\}$, where $\{x\}$ is the fractional part of $x$.

He has been good enough to let me see his manuscript and I am much obliged to him for this. His proof is very simple and really of an arithmetical nature. I give here a proof which is perhaps more direct and of an analytical character more appropriate for such questions. His proof makes use of the uniform distribution of $\{n \alpha\}$ where $\alpha$ is irrational, but it suffices for mine that $\{n \alpha\}$ takes an infinity of values for integer values of $n$.

I prove a slightly more general

THEOREM. Let $\alpha$ be a real number and let $f(x, y)$ be a polynomial in $x, y$ of degree $\geqq 1$ in $y$. Then

$$
F(x)=\sum_{n=0}^{\infty} f(n,\{n \alpha\}) x^{n},
$$

is a rational function of $x$ if and only if $\alpha$ is irrational.

On replacing $[n \alpha]$ by $n \alpha-\{n \alpha\}$ in (1), it suffices to prove the result for, say,

$$
F(x)=\sum_{n=0}^{\infty} f(n,\{n \alpha\}) x^{n},
$$

Hecke in his paper Ueber analytische Funktionen und die Verteilung von Zahlen mod Eins, Abh. Math. Sem. Univ. Hamburg vol. 1 (1921) pp. 54-76 proves the result when in (1), $f[n \alpha]=[n \alpha]$, in an

Received by the editors August 1, 1960.

1 Proc. Amer. Math. Soc. vol. 11 (1960) pp. 699-702. (Ed. note.) 
entirely different way by using results of Weyl on uniform distribution.

Questions involving polynomials in $\{n \alpha\}$ naturally suggest the Bernoulli polynomials. These are defined by

$$
\frac{z e^{z x}}{e^{z}-1}=\sum_{n=0}^{\infty} \frac{B_{n}(x)}{n !} z^{n}
$$

and so $B_{n}(x)$ is a polynomial of degree $n$ in $x$ whose expansion begins with $x^{n}-(1 / 2) n x^{n-1}+\cdots$. Also $B_{0}(x)=1, B_{1}(x)=x-1 / 2$. On multiplying (4) by $e^{z}-1$ and equating coefficients of $z^{n+1}$ on both sides, we find

$$
x^{n}=\sum_{r=0}^{n} \frac{n !}{r !(n-r+1) !} B_{r}(x) .
$$

We apply this to the powers of $\{n \alpha\}$ in (3), which then takes the form

$$
F(x)=\sum_{n=0}^{\infty}\left(\sum_{r, s=0}^{p} c_{r, s} n^{r} B_{s}(\{n \alpha\})\right) x^{n},
$$

say, where $r, s$ run through all non-negative integer values $\leqq p$ a constant integer independent of $n$, and the $c_{r s}$ are constants independent of $n$.

The series (1), (2), (3), (6) converge absolutely for $|x|<1$. Suppose first that $\alpha$ is rational, say $\alpha=p \mid q$ where $q$ is a positive integer. Then $\{n \alpha\}$ is a periodic function of $n$ with period $q$, and obviously $F(x)$ is a rational function of $x$ since $\sum_{n=0}^{\infty}\left(q n+q_{1}\right)^{t} x^{n}$ is clearly one when $t$ is a non-negative integer and $q_{1}$ is a constant.

Suppose next that $\alpha$ is irrational. We shall show that the circle $|x|=1$ is a line of essential singularities for $F(x)$ in (6), since the points $x=e^{2 m \pi i \alpha}$ for all integers $m$ except a finite number, are singularities of $F(x)$.

We have the well-known Fourier expansion for $s \geqq 0$

$$
B_{s}(\{y\})=\sum_{m=-\infty}^{\infty} d_{m, s} e^{-2 m \pi i y},
$$

where when $s>0, d_{m, s}=0$ if $m=0, d_{m, s}=(-1)^{s-1} s ! /(2 m \pi i)^{s}$ if $m \neq 0$. The series (7) converges absolutely except when $s=1$, and then $\sum_{-\infty}^{\infty}$ stands for $\lim _{N \rightarrow \infty} \sum_{m=-N}^{N}$. The result is easily obtained from (4). Thus

$$
d_{m, 8}=\int_{0}^{1} B_{s}(x) e^{2 m \pi i x} d x
$$


and so (4) gives

$$
\sum_{s=0}^{\infty} \frac{d_{m, s}}{s !} z^{s}=\int_{0}^{1} \frac{z e^{(2 m \pi i+z) x} d x}{e^{z}-1}=\frac{z}{2 m \pi i+z} .
$$

The result follows on equating coefficients of $z^{s}$ on both sides.

It may simplify the exposition if we consider first the special case when $c_{r s}=0$ for all $r>0$ in (6). We then put

$$
G(x)=\sum_{n=0}^{\infty}\left(\sum_{s=1}^{p} c_{s} B_{s}(\{n \alpha\})\right) x^{n}
$$

We deal first with the part of this arising when $s>1$. We substitute for $B_{s}(\{n \alpha\})$ from (7) in (8) which becomes an absolutely convergent double series. Sum for $n$, whence

$$
\sum_{n=0}^{\infty} B_{s}(\{n \alpha\}) x^{n}=\frac{(-1)^{s} s !}{(2 \pi i)^{s}} \sum_{m=-\infty}^{m=\infty} \frac{1}{m^{s}\left(1-x e^{-2 m \pi i \alpha}\right)},
$$

where the dash denotes the omission of the term with $m=0$. We considered more generally the series

$$
H(x)=\sum_{m=-\infty}^{\infty} \frac{b_{m}}{1-x e^{-2 m \pi i \alpha}},
$$

where the $b$ 's are independent of $x$ and only a finite number of the $b$ 's are zero. Suppose that the series $\sum b_{m}$ converges absolutely. We prove that $|x|=1$ is a line of essential singularities for $H(x)$, and that $x=e^{-2 \lambda x i \alpha}$ for all integers $\lambda$ except a finite number, is a singularity of $H(x)$. On putting $x=e^{-2 \lambda x i \alpha y}$ and then replacing $m$ by $m-\lambda$, it suffices to prove that if $b_{0}=1$, then $x=1$ is a singular point of $H(x)$. We show in fact that

$$
H(x) \sim \frac{1}{1-x} \quad \text { for } 0<x<1, x \rightarrow 1
$$

Write (10) as

$$
\begin{aligned}
H(x) & =\frac{1}{1-x}+\sum_{m=-N}^{N} \frac{b_{m}}{1-x e^{-2 m \pi i \alpha}}+\sum_{|m|>N} \frac{b_{m}}{1-x e^{-2 m \pi i \alpha}} \\
& =\frac{1}{1-x}+H_{1}(x)+H_{2}(x)=\frac{1}{1-x}+R(x),
\end{aligned}
$$

say. Now 


$$
\begin{aligned}
\left|1-x e^{-2 m \pi i \alpha}\right|^{2} & =1-2 x \cos 2 m \pi \alpha+x^{2}>(1-x)^{2} \\
& =(x-\cos 2 m \pi \alpha)^{2}+\sin ^{2} 2 m \pi \alpha \geqq \sin ^{2} 2 m \pi \alpha .
\end{aligned}
$$

Take $N$ so great that for given $\epsilon>0, \sum_{|m|>N}\left|b_{m}\right|<\epsilon$. Then

$$
\left|H_{2}(x)\right|<\frac{\epsilon}{1-x}, \quad\left|H_{1}(x)\right|<\sum_{m=-N}^{N} \frac{\left|b_{m}\right|}{|\sin 2 n \pi \alpha|}<M,
$$

where $M$ is independent of $x$. Hence

$$
|R(x)|<\frac{\epsilon}{1-x}+M
$$

and so for real $x$ with $0<x<1$ and $x \rightarrow 1$,

$$
H(x) \sim \frac{1}{1-x} .
$$

Consider next the term with $s=1$ in (7). Now

$$
B_{1}(\{y\})=\sum_{m=-\infty}^{\infty} \frac{e^{-2 m \pi i y}}{2 m \pi i}
$$

does not converge absolutely, and so we must proceed rather differently. Write

$$
\begin{aligned}
H_{0}(x) & =\sum_{n=0}^{\infty} B_{1}(\{n \alpha\}) x^{n} \\
& =\sum_{n=0}^{\infty}\left(\sum_{m=-\infty ; m \neq 0}^{\infty} \frac{e^{-2 m n \pi i \alpha}}{2 m \pi i}\right) x^{n}
\end{aligned}
$$

Let $\lambda$ be any integer $\neq 0$. Then

$$
\begin{aligned}
H_{0}\left(x e^{-2 \pi i \lambda \alpha}\right) & =\sum_{n=0}^{\infty}\left(\sum_{m=-\infty ; m \neq 0}^{\infty} \frac{e^{-2(m+\lambda) n \pi i \alpha}}{2 m \pi i}\right) x^{n} \\
& =\sum_{n=0}^{\infty}\left(\sum_{m=-\infty ; m \neq \lambda}^{\infty} \frac{e^{-2 m n \pi i \alpha}}{2(m-\lambda) \pi i}\right) x^{n}
\end{aligned}
$$

on changing $m$ into $m-\lambda$ which is permissible for the conditionally convergent series. Write

$$
\begin{aligned}
& G_{0}(x)=H_{0}(x)-H_{0}\left(x e^{-2 \pi i \lambda \alpha}\right) \\
& (12)=\sum_{n=0}^{\infty}\left(\sum_{m=-\infty ; m \neq 0, \lambda}^{\infty} \frac{-\lambda e^{-2 m n \pi i \alpha}}{2 m(m-\lambda) \pi i}\right) x^{n}+\sum_{n=0}^{\infty} \frac{e^{-2 \lambda n \pi i \alpha}}{2 \lambda \pi i} x^{n}+\sum_{n=0}^{\infty} \frac{x^{n}}{2 \lambda \pi i} .
\end{aligned}
$$


The two simple series on the right have simple poles at $x=e^{2 \lambda \pi i \alpha}$ and $x=1$ respectively. The double series on the right is absolutely convergent, and summing for $n$, it becomes, say

$$
G_{1}(x)=\sum_{m=-\infty ; m \neq 0, \lambda}^{\infty} \frac{-\lambda}{2 m(m-\lambda) \pi i} \frac{1}{1-x e^{-2 m \pi i \alpha}} .
$$

Since $\sum 1 / m(m-\lambda)$ converges absolutely, the series defines a function of $x$ with $|x|=1$ as a line of essential singularities. Also for $m \neq 0, \lambda$,

$$
G_{1}(x) \sim \frac{-\lambda}{2 m(m-\lambda) \pi i} \frac{1}{1-x e^{-2 m \pi i \alpha}}
$$

for $0<x e^{-2 m \pi i \alpha}<1$, and $x e^{-2 m \pi i \alpha} \rightarrow 1-0$. We have now from (8), for $m \neq 0, \lambda$,

$$
G(x)-G\left(x e^{-2 \lambda x i \alpha}\right) \sim \frac{c}{1-x e^{-2 m \pi i \alpha}},
$$

where

$$
c=\sum_{s=1}^{p} \frac{c_{s} s !(-1)^{s}}{(2 \pi i)^{s}}\left(\frac{1}{m^{s}}-\frac{1}{(m-\lambda)^{s}}\right) .
$$

Suppose now that for some $m \neq 0, \lambda, x=e^{2 m \pi i \alpha}$ is not a singular point of the left hand side of (13). Then $c=0$, and then (14) gives a polynomial equation in $m$ and so only a finite number of values of $m$. Excluding these, $x=e^{2 m \pi i \alpha}$ is singular for either $G(x)$ or $G\left(x e^{-2 \lambda \pi i \alpha}\right)$. Hence if there is one $m$ not in this excluded set for which $G(x)$ is not singular, then $G(x)$ is singular for $x=e^{2(\lambda+m) \pi i \alpha}$. Since $\lambda$ is arbitrary except that $\lambda \neq m, G(x)$ has singularities everywhere dense on $|x|=1$.

We consider finally (6) when $r>0$. It is obvious that as $x \rightarrow 1$, $\sum_{n=1}^{\infty} n^{r} x^{n} \sim k(1-x)^{-r-1}$ where $k$ is a constant. This means that when (6) is transformed as before, the singularity at $x=e^{2 m \pi i \alpha}$ is dominated by the term with $r=p$, i.e. by $\left(1-x e^{-2 m \pi i \alpha}\right)^{p+1}$. The argument proceeds exactly as before except that the denominators $1-x e^{-2 m \pi i \alpha}$ are replaced by $\left(1-x e^{-2 m \pi i \alpha}\right)^{p+1}$.

This completes the proof.

I should like to thank Professor Davenport for his comments on my manuscript.

St. John's College, Cambridge, England 